\title{
IDENTIFYING THE CANONICAL COMPONENT FOR THE WHITEHEAD LINK
}

\author{
EMiLY LANDES
}

ABstract. In this paper, we determine topologically the canonical component of the $S L_{2}(\mathbb{C})$ character variety of the Whitehead link complement.

\section{Introduction}

Since the seminal work of Culler and Shalen, character varieties have proven to be a powerful tool for studying hyperbolic 3-manifolds; for exampe, they provide efficient means of detecting essential surfaces in hyperbolic knot complements $[3,2,9]$. For such a useful tool, the character variety itself is a rather basic concept and yet determining explicit models for even the simplest hyperbolic knot complements, let alone link complements, is a difficult problem. We are particularly interested in the canonical component (i.e. that containing a character of a discrete faithful representation) of these character varieties. Only recently have explicit models for the canonical components of a full family of hyperbolic knots been determined [7].

In beginning to understand the explicit models for hyperbolic knots, our attention extends to constructing canonical components of hyperbolic two component links. In [7] the authors determine the $S L_{2} \mathbb{C}$ character varieties for the twist knots. As the twist knots can be obtained by Dehn filling one of the cusps of the Whitehead link complement, we are naturally interested in constructing the particular character variety of the Whitehead link and studying the effect Dehn surgery has on the character variety. The focus of this paper is to construct the canonical component for the $S L_{2} \mathbb{C}$ character variety of the Whitehead link complement, which we will do as the following theorem.

Theorem 1. The canonical component of the character variety of the Whitehead link complement is a rational surface isomorphic to $\mathbb{P}^{2}$ blown up at ten points.

Understanding an algebro-geometric surface usually means understanding a surface up to birational equivalence. One reason for this is that a lot of the information about a variety is carried by the birational equivalence class. For complex curves, the birational equivalence class contains a unique smooth model (up to isomorphism). However, for complex surfaces, although there may be more than one smooth model in a birational equivalence class, there is a notion of a minimal smooth model. That is the smooth birational model which has no $(-1)$ curves.

The minimal smooth model for the canonical component of the Whitehead link is $\mathbb{P}^{2}$. We are able to determine this minimal model from a particular projective model, $S$, for the canonical component. The defining polynomial for the canonical component

Received by the editors November 11, 2010. Revision received January 31, 2011. 
of the Whitehead link cuts out an affine surface in $\mathbb{C}^{3}$. Compactifying this surface in $\mathbb{P}^{2} \times \mathbb{P}^{1}$ gives the singular surface, $S$. Although $S$ is a singular surface, it is birational to a conic bundle, which in turn is birational to $\mathbb{P}^{2}$.

There are three models for the components of the algebraic sets we discuss in this paper. There is the affine model defined by an ideal of polynomials in $\mathbb{C}^{3}$. There is the projective model, which may or may not be smooth, obtained by compactifying the affine model in $\mathbb{P}^{2} \times \mathbb{P}^{1}$. Finally, if the projective model is not smooth, there is the smooth projective model obtained resolving singular points of the projective model. In this paper when we refer to components of the character variety, we mean the smooth projective model. We will specify when speaking of an affine model or a singular projective model.

Although knowing the birational equivalence class is helpful in understanding how the variety behaves, determining the variety topologically requires understanding the isomorphism class. In this case, since $S$ is rational, we can use the minimal model to determine the isomorphism class. Smooth surfaces birational to $\mathbb{P}^{2}$ are isomorphic either to $\mathbb{P}^{1} \times \mathbb{P}^{1}$ or to $\mathbb{P}^{2}$ blown up at $n$ points. For smooth surfaces, this isomorphism class can be determined directly from the Euler characteristic. Although we can calculate the Euler characteristic for $S$, it does not determine the isomorphism class since $S$ is not smooth. Rather than work with the singular surface $S$ we will work with the smooth surface obtained by resolving the singularities of $S$.

Away from the four singular points, $S$ looks like a conic bundle in the sense that it is a bundle over $\mathbb{P}^{1}$ whose fibers are conics, i.e., curves in $\mathbb{P}^{2}$ cut out by degree 2 polynomials. While the total space of a conic bundle is smooth a given fiber may not be. The model, $S$, for the canonical component of the Whitehead link has six fibers which are not smooth. Five of these fibers are degenerate i.e. have exactly one singularity, whereas the sixth fiber is a double line, i.e., every point is a singularity. It is worth remarking that double lines are a fairly rare feature to conic bundles. More precisely, all conics can be parameterized by $\mathbb{P}^{5}$ and the double lines correspond to a codimension three subvariety [4]. Hence a conic bundle with a double line fiber corresponds to a line that passes through a particular codimension 3 subvariety in $\mathbb{P}^{5}$ which is a rare occurrence.

Since $S$ is birational to a conic bundle and so birational to $\mathbb{P}^{2}$, it is isomorphic either to $\mathbb{P}^{1} \times \mathbb{P}^{1}$ or $\mathbb{P}^{2}$ blown up at $n$ points. For $n \leq 8$, the surfaces $\mathbb{P}^{2}$ blown up at $n$ points are nice algebro-geometric objects in the sense that they exhibit only finitely many $(-1)$ curves, that is curves with self-intersection number -1 . The canonical component of the Whitehead link, $\mathbb{P}^{2}$ blown up at ten points, has infintely many $(-1)$ curves.

The Whitehead link complement can be obtained by $1 / 1$ Dehn surgery on the Borromean rings (the complement of which we will denote by $M_{\mathrm{br}}$ ). The manifold which results upon $1 / n$ Dehn filling on one of the cusps of $M_{\mathrm{br}}$ is a hyperbolic two component 2-bridge link complement [6]. For $n=1, \ldots, 4$, we were able to use Mathematica to determine the polynomials which define the character varieties of $M_{\mathrm{br}}(1 / n)$. For $n=1, \ldots, 4$, the character variety of $M_{\mathrm{br}}(1 / n)$ has a component which is a rational surface. Aside from the Whitehead link, the rational component(s) of these character varieties are not canonical components.

Theorem 2. For $n=2,3,4$, the character variety of $M_{b r}(1 / n)$ has a component which is a rational surface isomorphic to $\mathbb{P}^{2}$ blown up at 7 points. 
Similar to the canonical component of the Whitehead link, all of these rational surfaces exhibit a double line fiber. Unlike the Whitehead link, these rational surfaces have finitely many $(-1)$ curves. The surface $\mathbb{P}^{2}$ blown up at 7 points has exactly $47(-1)$ curves. There is something to be said for the fact that examples of this surface come from canonical components of character varieties of hyperbolic link complements. It is a start to understanding how topology and algebraic geometry fit together.

We start, in Section 2, by defining the character variety. In Section 3, we provide some background in algebraic geometry. The main theorem will be proved in Section 4. In Section 5, we discuss the character varieties for similar hyperbolic two component 2-bridge link complements.

\section{Preliminaries}

Here we briefly describe the $S L_{2}(\mathbb{C})$ representation and character varieties. Standard references on this include [3] and [9].

2.1. Representation variety. For any finitely generated group $\Gamma=\left\langle g_{1}, \ldots, g_{n} \mid r_{1}, \ldots, r_{m}\right\rangle$, the set of $S L_{2}(\mathbb{C})$ representations $R(\Gamma)=\operatorname{Hom}\left(\Gamma, S L_{2} \mathbb{C}\right)$ has the structure of an affine algebraic set [3]. We view this space as $R(\Gamma)=$ $\left\{\left(x_{1}, \ldots, x_{n}\right) \in\left(S L_{2}(\mathbb{C})\right)^{n} \mid r_{j}\left(x_{1}, \ldots, x_{n}\right)=I, j=1, \ldots, m\right\}$. Notice that $R(\Gamma)$ can be identified with $r^{-1}(I, \ldots, I)$ where $r:\left(S L_{2}(\mathbb{C})\right)^{n} \rightarrow\left(S L_{2}(\mathbb{C})\right)^{m}$ is the map $r(x)=\left(r_{1}(x), \ldots, r_{m}(x)\right)$. That $R(\Gamma)$ is an algebraic set follows from the fact that $r$ is a regular map. Identifying $S L_{2}(\mathbb{C})^{n}$ with a subset of $\mathbb{C}^{4 n}$, we can view $R(\Gamma)$ as an algebraic set over $\mathbb{C}$. We should note that the isomorphism class of $R(\Gamma)$ does not depend on the group presentation and in general, $R(\Gamma)$ is not irreducible. In fact, the abelian representations (i.e. representations with abelian image) comprise a component of this algebraic set [3].

2.2. Character variety. The character of a representation $\rho: \Gamma \rightarrow S L_{2}(\mathbb{C})$ is a map $\chi_{\rho}: \Gamma \rightarrow \mathbb{C}$ defined by $\chi_{\rho}(\gamma)=\operatorname{tr}(\rho(\gamma))$. The set of characters is defined to be $X(\Gamma)=\left\{\chi_{\rho} \mid \rho \in R(\Gamma)\right\}$. For each $g \in \Gamma$ there is a regular map $\tau_{g}: R(\Gamma) \rightarrow \mathbb{C}$ defined by $\tau_{g}(\rho)=\chi_{\rho}(g)$. Let $T$ be the subring of the coordinate ring on $R(\Gamma)$ generated by 1 and $\tau_{g}, g \in \Gamma$. In [3] it is shown that the ring $T$ is finitely generated, for example by $\left\{\tau_{g_{i_{1}} g_{i_{2}} \ldots g_{i_{k}}} \mid 1 \leq i_{1}<i_{2}<\cdots<i_{k} \leq n\right\}$. In particular any character $\chi \in X(\Gamma)$ is determined by its value on finitely many elements of $\Gamma$. As a result, for $t_{1}, \ldots, t_{s}$ generators of $T$, the map $t=\left(t_{1}, \ldots, t_{s}\right): R(\Gamma) \rightarrow \mathbb{C}^{s}$ defined by $\rho \mapsto\left(t_{1}(\rho), \ldots, t_{s}(\rho)\right)$ induces a map $X(\Gamma) \rightarrow \mathbb{C}^{s}$. Culler and Shalen use the fact that this map is injective to show that $X(\Gamma)$ inherits the structure of an algebraic set [3].

Let $M$ be a hyperbolic manifold and let $\Gamma=\pi_{1}(M)$. We refer to the affine algebraic set $\tilde{X}\left(\pi_{1}(M)\right)$ as the affine $S L_{2}(\mathbb{C})$ character variety of $M$. The affine canonical component of $\tilde{X}\left(\pi_{1}(M)\right)$ is the component containing a character, $\chi_{0}$, of a discrete faithful representation and is denoted by $\tilde{X}_{0}\left(\pi_{1}(M)\right)$. We are interested not so much in the affine model but in a closed projective model. By $S L_{2}(\mathbb{C})$ character variety and canonical component we mean the projective models $X\left(\pi_{1}(M)\right)$ and $X_{0}\left(\pi_{1}(M)\right)$ respectively. For hyperbolic knots and links as $\chi_{0}$ is a smooth point, $X_{0}$ is unique [10]. In this context, Thurston's Hyperbolic Dehn Surgery Theorem states that for 
an orientable, hyperbolic 3 -manifold of finite volume, with $n$-cusps, $X_{0}$ has complex dimension $n$.

We will be particularly interested in studying $X_{0}$ for hyperbolic two component 2-bridge link complements. These will be complex surfaces. A more detailed account of the character variety in this case will be discussed in Section 4 .

\section{Algebraic geometry}

The purpose of this section is to review the algebro-geometric concepts relevant to the main proof of this paper. For more details see [5] or [8].

3.1. Conic bundles. The character varieties of all of our examples have a component which is a conic bundle. A conic is a curve defined by a polynomial over $\mathbb{P}^{2}$ of degree 2. Smooth conics have the genus zero [8] so are spheres. A degenerate conic consists of two spheres intersecting one one point. In this paper, the term conic bundle will be used to mean a conic bundle over $\mathbb{P}^{1}$, i.e., over a sphere. Conic bundles are nice algebro-geometric objects. Whilst there is no classification of complex surfaces, there is a classification for the subclass of $\mathbb{P}^{1}$ bundles over $\mathbb{P}^{1}$, which are slightly different than conic bundles in the sense that conic bundles may can have fibers with singularities. Any $\mathbb{P}^{1}$ bundle over $\mathbb{P}^{1}$ comes from a projectivized rank-2 vector bundle over $\mathbb{P}^{1}$. As the rank- 2 vector bundles are parametrized by $\mathbb{Z}$, the $\mathbb{P}^{1}$ bundles over $\mathbb{P}^{1}$ are parameterized by $\mathbb{Z}$. Each vector bundle over $\mathbb{P}^{1}$ can be written as $E=\mathbb{O} \oplus \mathbb{O}(-e)[1,5]$. Here $\mathbb{O}$ denotes the trivial rank-2 vector bundle over $\mathbb{P}^{1}$ and $\mathbb{O}(-e)$ denotes the vector bundle whose section has self-intersection number $e$.

Proposition 1. A conic bundle is a rational surface.

Proof. Any conic bundle $T$ can be realized as a hypersurface defined by a polynomial $f_{T}$ of bidegree $(2, m)$ over $\mathbb{P}^{2} \times \mathbb{P}^{1}$. In particular, a generic fiber of the coordinate projection of $T$ to $\mathbb{P}^{1}$ is a nondegenerate conic. This means that $T$ is locally, and hence birationally, equivalent to $\mathbb{P}^{1} \times \mathbb{P}^{1}$, which is birationally equivalent to $\mathbb{P}^{2}$.

Another way to see that $T$ is rational is by looking at the canonical divisor. The canonical divisor $K_{T}$ of $T$ is the canonical divisor $K_{\mathbb{P}^{2} \times \mathbb{P}^{1}}$ of $\mathbb{P}^{2} \times \mathbb{P}^{1}$ twisted by the divisor class of $T$, all restricted to $T$. Namely $K_{T}=\left.\left(\mathcal{O}_{\mathbb{P}^{2} \times \mathbb{P}^{1}}(-3,-2) \otimes \mathcal{O}_{\mathbb{P}^{2} \times \mathbb{P}^{1}}(2, m)\right)\right|_{S}=$ $\left.\mathcal{O}_{\mathbb{P}^{2} \times \mathbb{P}^{1}}(-1, m-2)\right|_{S}$. In particular, the canonical divisor $K_{T}$ corresponds to the line bundle $\left.\mathcal{O}_{\mathbb{P}^{2} \times \mathbb{P}^{1}}(-1, m-2)\right|_{S}$ the number of global sections of which are characterized by the number of polynomials of bidegree $(-1, m-2)$. Since there are no polynomials of bidegree $(-1, m-2)$ there are no global sections on $T$. The only surfaces in which the canonical bundle has no global sections are rational and ruled (i.e., birational to $\mathbb{P}^{2}$ and a fibration over a curve with $\mathbb{P}^{2}$ fibers).

Corollary 1. A conic bundle is isomorphic to either $\mathbb{P}^{1} \times \mathbb{P}^{1}$ or $\mathbb{P}^{2}$ blown up at $n$ points for some integer $n$.

It is a known fact ([5] Chapter V) that for two birational varieties the birational equivalence between them can be written as sequence of blow ups and blow downs. In particular, $\mathbb{P}^{2}$ is birational to either $\mathbb{P}^{1} \times \mathbb{P}^{1}$ or to $\mathbb{P}^{2}$ blown up at $n$ points. Hence, any rational surface is isomorphic to $\mathbb{P}^{1} \times \mathbb{P}^{1}$ or to $\mathbb{P}^{2}$ blown up at $n$ points. 
3.2. Blow ups. Blowing up varieties at points is a standard tool for resolving singularities and determining isomorphism classes of surfaces and we make repeated use of such in this paper.

Since blowing up is a local process, we can do all of our blow ups in affine neighborhoods. For our purposes, understanding what it means to blow up subvarieties of $\mathbb{A}^{2}$ and $\mathbb{A}^{3}$ at a point should be sufficient. For more details refer to [5] or [8].

Intuitively blowing up $\mathbb{A}^{2}$ at a point can be described as replacing a point in $\mathbb{A}^{2}$ by an exceptional divisor (i.e., a copy of $\mathbb{P}^{1}$ ). To understand this more concretely, we will describe the blow up of $\mathbb{A}^{2}$ at the origin. Consider the product $\mathbb{A}^{2} \times \mathbb{P}^{1}$. Take $x, y$ as the affine coordinates of $\mathbb{A}^{2}$ and $t, u$ as the homogeneous coordinates of $\mathbb{P}^{1}$. The blow up of $\mathbb{A}^{2}$ at $(0,0)$ is the closed subset $Y=\{[x, y: t, u] \mid x u=t y\}$ in $A^{2} \times \mathbb{P}^{1}$. The blow up comes with a natural map $\gamma: Y \rightarrow \mathbb{A}^{2}$, which is just projection onto the first factor. Notice that the fiber over any point $(x, y) \neq(0,0) \in \mathbb{A}^{2}$ is precisely one point in $Y$. However, the fiber over $(x, y)=(0,0)$, is a $\mathbb{P}^{1}$ worth of points in $Y$ (i.e. $\{(0,0, t, u)\} \subset Y)$. Since $\mathbb{A}^{2}-\{(0,0)\} \simeq Y-\gamma^{-1}(0,0), \gamma$ is a birational map and $\mathbb{A}^{2}$ is birational to $Y$. Blowing up $\mathbb{A}^{2}$ at a point $p \neq 0$ simply amounts to a change in coordinates.

Suppose we want to blow up a subvariety $X \subset \mathbb{A}^{2}$ at a point, $p$. Take the blow up $Y$ of $\mathbb{A}^{2}$ at $p$. Then the blow up $\left.B l\right|_{p}(X)$ of $X$ at $p$ is the closure $\overline{\gamma^{-1}(X-p)}$ in $Y$ where $\gamma$ is as described above. We note that $\left.B l\right|_{p}(X)$ is birational to $X-p$ and if $\left.B l\right|_{p}(X)$ is smooth, $\gamma^{-1}(p)$ will intersect $Y$ in a zero dimensional variety.

For our paper, we need to understand how blowing up a surface at a smooth point affects the Euler characteristic.

Proposition 2. The Euler characteristic of a surface $X$ blown up at a smooth point $p$ is $\chi\left(\left.B l\right|_{p}(X)\right)=\chi(X)+1$.

Proof. To blow up $X$ at a smooth point $p$ we work locally in an affine neighborhood about $p$. Near $p, X$ is locally $\mathbb{A}^{2}$ near 0 . Hence the result of blowing up $X$ at $p$ is the same as blowing up $\mathbb{A}^{2}$ at 0 . In terms of the Euler characteristic this amounts to replacing a point with an exceptional $\mathbb{P}^{1}$. In particular, $\chi\left(B l_{p}(X)\right)=\chi(X-\{p\})+$ $\chi\left(\mathbb{P}^{1}\right)=\chi(X)+1$.

In order to resolve singularities we will need to blow up subvarieties of $\mathbb{A}^{3}$ at a point. Taking $x_{1}, x_{2}, x_{3}$ as affine coordinates for $\mathbb{A}^{3}$ and $y_{1}, y_{2}, y_{3}$ as projective coordinates for $\mathbb{P}^{2}$, the blow up of $A^{3}$ at the origin is the closed subvariety, $Y^{\prime}=$ $\left\{\left[x_{1}, x_{2}, x_{3}: y_{1}, y_{2}, y_{3}\right] \mid x_{1} y_{2}=x_{2} y_{1}, x_{1} y_{3}=x_{3} y_{1}, x_{2} y_{3}=x_{3} y_{2}\right\}$ in $\mathbb{A}^{3} \times \mathbb{P}^{2}$. Just as in the case of $\mathbb{A}^{2}$, this blow up comes with a natural map $\gamma: Y^{\prime} \rightarrow \mathbb{A}^{3}$ which is simply projection onto the first factor. Just as before, the fiber over any point $\left(x_{1}, x_{2}, x_{3}\right) \neq(0,0,0) \in \mathbb{A}^{3}$ is precisely one point in $Y^{\prime}$. However, the fiber over $\left(x_{1}, x_{2}, x_{3}\right)=(0,0,0)$, is a $\mathbb{P}^{2}$ worth of points in $Y^{\prime}$ (i.e., $\left.\left\{\left(0,0,0, y_{1}, y_{2}, y_{3}\right)\right\} \subset Y^{\prime}\right)$. Since $\mathbb{A}^{3}-\{(0,0,0)\} \simeq Y^{\prime}-\gamma^{-1}(0,0,0), \gamma$ is a birational map and $\mathbb{A}^{3}$ is birational to $Y^{\prime}$. Blowing up $\mathbb{A}^{3}$ at a point $p \neq 0$ simply amounts to a change in coordinates. To blow up a subvariety $X \subset \mathbb{A}^{3}$ at a point $p$, first take the blow up $Y^{\prime}$ of $\mathbb{A}^{3}$ at $p$. Then the blow up $\left.B l\right|_{p}(X)$ of $X$ at $p$ is the closure $\overline{\gamma^{-1}(X-p)}$ in $Y^{\prime}$. We note that $\left.B l\right|_{p}(X)$ is birational to $X-p$ and if $\left.B l\right|_{p}(X)$ is smooth, $\gamma^{-1}(p)$ will intersect $Y^{\prime}$ in a smooth curve. 
In this paper, we obtain smooth surfaces by resolving singularities. As the Euler characteristic of these smooth surfaces helps us determine the isomorphism class we keep track of how blowing up singular points affects the Euler characteristic.

Proposition 3. If the blow up $\left.B l\right|_{p}(X)$ of a surface $X$ at a singular point $p$ is smooth, then the Euler characteristic of $\left.B l\right|_{p}(X)$ is $\chi\left(\left.B l\right|_{p}(X)\right)=\chi(X)+2 g+1$ where $g$ is the genus of the curve $\gamma^{-1}(p)$ in $\left.B l\right|_{p}(X)$.

Proof. Away from the point $p, X$ is isomorphic to $B l_{p}(X) \backslash \gamma^{-1}(p)$. Hence, $\chi\left(B l_{p}(X)\right)=$ $\chi(X-p)+\chi\left(\gamma^{-1}(p)\right)$. The preimage $\gamma^{-1}(p)$ in $B l_{p}(X)$ is a smooth codimension 1 subvariety of the fiber over $p$ in $B l_{p}\left(A^{3}\right)$. Since the fiber over $p$ in $B l_{p}\left(A^{3}\right)$ is a $\mathbb{P}^{2}$, $\gamma^{-1}(p)$ in $B l_{p}(X)$ is a smooth curve of genus $g$. Hence $\chi\left(\gamma^{-1}(p)\right)=2 g+2$ and $\chi\left(B l_{p}(X)\right)=\chi(X-\{p\})+\chi\left(\gamma^{-1}(p)\right)=\chi(X)+2 g+1$.

3.3. Total transformations. For the proof of Proposition 5, we will use a total transform to extend a map $\phi$ between projective varieties. The description we provide here comes from [5, p 410]. We begin by setting up some notation. Let $X$ and $Y$ be projective varieties.

Definition 1. A birational transformation $T$ from $X$ to $Y$ is an open subset $U \subset X$ and a morphism $\phi: U \rightarrow Y$ which induces an isomorphism on the function fields of $\mathrm{X}$ and $\mathrm{Y}$.

Since different maps must agree on the overlap for different open sets, we take the largest open set $U$ for which there is such a morphism $\phi$. It is common to say that $T$ is defined at the points of $U$ and

Definition 2. The fundamental points of $T$ are those in the set $X-U$.

For $G$ the graph of $\phi$ in $U \times Y$, let $\bar{G}$ be the closure of $G$ in $X \times Y$. Let $\rho_{1}: \bar{G} \rightarrow X$ and $\rho_{2}: \bar{G} \rightarrow Y$ be projections onto the first and second factors respectively.

Definition 3. For any subset $Z \subset X$ the total transform of $Z$ is $T(Z):=\rho_{2}\left(\rho_{1}^{-1}(Z)\right)$.

For a point $p \in U, T(p)$ is consistent with $\phi(p)$; while for a point $p \in X-U, T(p)$ is generally larger than a single point (in our examples it will be a copy of $\mathbb{P}^{1}$ ).

3.4. Intersection numbers of curves. A smooth curve $C$ in $\mathbb{P}^{1} \times \mathbb{P}^{1}$ is cut out by a polynomial $g$ which is homogenous in each of the $\mathbb{P}^{1}$ coordinates. We say $g$ has bidgree $(a, b)$ where $a$ is the degree of $g$ viewed as polynomial over the first factor and $b$ is the degree of $g$ viewed as a polynomial over the second factor. In the proof of Theorem 1, we will determine the number of intersections of two smooth curves in $\mathbb{P}^{1} \times \mathbb{P}^{1}$ based solely on the bidegrees of their defining polynomials. Suppose $C_{1}$ and $C_{2}$ are two smooth curves cut out by irreducible polynomials $g_{1}$ and $g_{2}$ of bidegrees $\left(a_{1}, b_{1}\right)$ and $\left(a_{2}, b_{2}\right)$, respectively. Counting multiplicities, $C_{1}$ and $C_{2}$ intersect in $a_{1} b_{2}+a_{2} b_{1}$ points [5, Section 5.1].

3.5. Geometric genus. The geometric genus, $p_{g}$, of a projective variety, $S$, is the dimension of the vector space of global sections $\Gamma\left(X, \omega_{k}\right)$ of the canonical divisor $w_{k}$. For a complex curve, the geometric genus coincides with the topological genus and can thus be used to topologically determine the character varieties of hyperbolic knot complements. Unfortunately for complex surfaces, the geometric genus does not 
carry as direct topological information (for instance it appears as $h^{2,0}$ in the Hodge decomposition [4]). However, as it may still be helpful in determining which varieties can arise as the character varieties of hyperbolic two component link complements, it it worth keeping track of this value. For a hypersuface, $Z$, in $\mathbb{P}^{2} \times \mathbb{P}^{1}$ defined by a polynomial $f$ of bidegree $(a, b)$ the geometric genus is $p_{g}(Z)=\frac{(a-1)(a-2)(b-1)}{2}$.

We give a brief description of this here. As the group of linear equivalence classes of divisors for $\mathbb{P}^{2} \times \mathbb{P}^{1}$ is $\operatorname{Pic}\left(\mathbb{P}^{2} \times \mathbb{P}^{1}\right) \cong \mathbb{Z} \times \mathbb{Z}$, we think of the divisors of $\mathbb{P}^{2} \times \mathbb{P}^{1}$ as elements of $\mathbb{Z} \times \mathbb{Z}$. For a linear class with representative divisor $D$ on $\mathbb{P}^{2} \times \mathbb{P}^{1}$, there is an associated vector space, $L(D)$ of principal divisors $E$ such that $\mathrm{D}+\mathrm{E}$ is effective. The vector space $L(D)$ is in one-to-one correspondence with the vector space of global sections of the line bundle $\mathbb{L}(D)$ on $\mathbb{P}^{2} \times \mathbb{P}^{1}$. As the vector space of global sections of $\mathbb{L}(D)$ corresponds to the space of polynomials over $\mathbb{P}^{2} \times \mathbb{P}^{1}$ with the same bidegree as that which cuts out $D$, the restrictions of these polynomials to $S$, which are nonzero on $S$, correspond to the vector space of global sections of $D$ on $S$. That is to say the kernel of the surjective map $\left.L(D) \rightarrow \mathrm{L}(D)\right|_{S}$ is those polynomials which vanish on $S$. When $D$ is the the canonical divisor $K_{S}$ of the surface $S$, assuming all the restricted polynomials are nonzero on $S$, the geometric genus of the surface $g_{g}(S)$ is then just the dimension of the vector space of these polynomials.

For the hypersurface $S$ defined by $f$, we can use the adjunction formula to determine $K_{S}$. Namely, $K_{S}=\left.\left[K_{\mathbb{P}^{2} \times \mathbb{P}^{1}} \otimes \mathbb{O}(S)\right]\right|_{S}$. The canonical divisor $K_{\mathbb{P}^{2} \times \mathbb{P}^{1}}$ of $\mathbb{P}^{2} \times \mathbb{P}^{1}$ is $(-3,-2) \in \mathbb{Z} \times \mathbb{Z}$ and the divisor class $\mathbb{O}(S)=(a, b) \in \operatorname{Pic}\left(\mathbb{P}^{2} \times \mathbb{P}^{1}\right) \cong \mathbb{Z} \times \mathbb{Z}$ since $f$ has bidegree $(a, b)$. Hence, $K_{S}=(a-3, b-2)$. Since the linear class of divisors of $K_{S}=(a-3, b-2)$ corresponds to a polynomials of bidegree $(a-3, b-2)$, the global sections of line bundle associated to $K_{S}=\left.(a-3, b-2)\right|_{S}$ correspond to polynomials of bidegree $(a-3, b-2)$. Since $S$ is a hypersuface defined by the irreducible polynomial $f$, no polynomial of bidegree $(a-3, b-2)$ can vanish on all of $S$. Hence, the geometric genus of the surface $g_{g}(S)$ is then just the dimension of the vector space of polynomials over $\mathbb{P}^{2} \times \mathbb{P}^{1}$ of bidegree $(a, b)$. Determining this dimension is a matter of counting monomials of bidegree $(a-3, b-3)$ for which there are $\frac{(a-1)(a-2)(b-1)}{2}$.

3.6. Projective models for character varieties. The affine varieties with which we are concerned are all hypersurfaces in $\mathbb{C}^{3}$, i.e., they are zero sets $Z(\tilde{f})$ of a single smooth polynomial $\tilde{f} \in \mathbb{C}[x, y, z]$. Finding the right projective completion is tricky, especially with complex surfaces since different projective completions may result in non-isomorphic models. It might seem natural to take projective closures in $\mathbb{P}^{3}$. One problem with compactifying in $\mathbb{P}^{3}$ is that, generally, this projective model has singularities that take more than one blow up to resolve. Following the work of [7] it is more natural to consider the compactification in $\mathbb{P}^{2} \times \mathbb{P}^{1}$. This compactification does result in a singular surface. However, the singularities are manageable and away from the singularities this model has the nice structure of a conic bundle. Hence, for these reasons, this is the projective model we choose to use for our examples.

Given an affine variety $Z(\tilde{f})$ defined by a polynomial $\tilde{f} \in \mathbb{C}[x, y, z]$, we construct the projective closure by homogenizing $\tilde{f}$. Let $a$ be the degree of $\tilde{f}$ when viewed as a polynomial in variables $x$ and $y$. Let $b$ be the degree of $\tilde{f}$ when viewed as a polynomial in the variable $z$. The projective model in $\mathbb{P}^{2} \times \mathbb{P}^{1}$ of the affine variety $Z(\tilde{f})$ is cut out by the homogeneous polynomial $f=u^{a} w^{b} \tilde{f}\left(\frac{x}{u}, \frac{y}{u}, \frac{z}{w}\right)$, where $x, y, u$ are $\mathbb{P}^{2}$ coordinates 
and $z, w$ are $\mathbb{P}^{1}$ coordinates. Notice that every monomial that appears in $f$ has degree $a$ in the $\mathbb{P}^{2}$ coordinates and degree $b$ in the $\mathbb{P}^{1}$ coordinates so $f$ has bidegree $(a, b)$.

\section{The Whitehead link}

Let $W$ denote the complement of the Whitehead link in $S^{3}$ and let $\Gamma_{W}=\pi_{1}(W)$. Then $\Gamma_{W}=\langle a, b \mid a w=w a\rangle$ where $w$ is the word $w=b a b^{-1} a^{-1} b^{-1} a b$ (Figure 1).

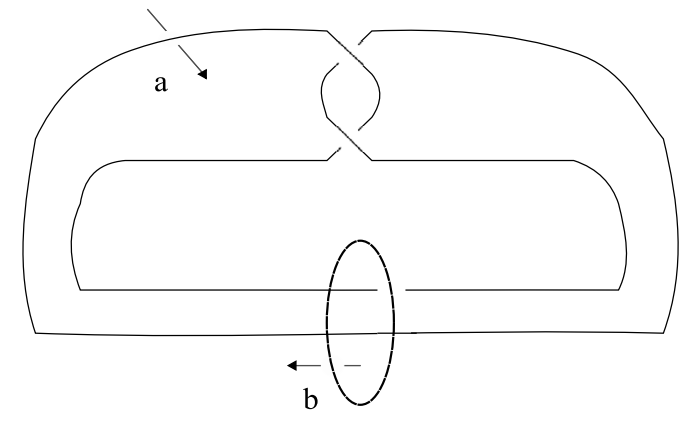

Figure 1. The Whitehead link.

Proposition 4. $\tilde{X}(W)$ is a hypersurface in $\mathbb{C}^{3}$.

Proof. To determine the defining polynomial for $\tilde{X}(W)$ in $\mathbb{C}^{3}$, we look at the image of $R(W)$ under the map $t=\left(t_{1}, \ldots, t_{s}\right): R(W) \rightarrow \mathbb{C}^{s}$ as defined in Section 2. We begin by establishing the defining ideal for $R(W)$. Any representation of $\rho \in R(W)$ can be conjugated so that

$$
\bar{a}=\rho(a)=\left(\begin{array}{cc}
m & 1 \\
0 & m^{-1}
\end{array}\right) \quad \bar{b}=\rho(b)=\left(\begin{array}{cc}
s & 0 \\
r & s^{-1}
\end{array}\right) .
$$

The polynomials that define $R(W)$ then come from the relation $\bar{w} \bar{a}-\bar{a} \bar{w}=0$. Writing $\rho(w)=\left(\begin{array}{ll}w_{11} & w_{12} \\ w_{21} & w_{22}\end{array}\right)$, we see that

$$
\bar{w} \bar{a}-\bar{a} \bar{w}=\left(\begin{array}{cc}
-w_{21} & w_{11}+w_{12}\left(m^{-1}-m\right)-w_{22} \\
w_{21}\left(m-m^{-1}\right) & w_{21}
\end{array}\right) .
$$

Hence, the representation variety is cut out by the ideal $\left\langle p_{1}, p_{2}\right\rangle \subset \mathbb{C}\left[m, m^{-1} r, s, s^{-1}\right]$ where $p_{1}=w_{21}$ and $p_{2}=w_{11}+w_{12}\left(m^{-1}-m\right)-w_{22}$.

For the Whitehead link

$$
\begin{aligned}
p_{1}= & m^{-2} s^{-2} r\left(r-m^{2} r+m s-m^{3} s+2 m r^{2} s-m^{3} r^{2} s-r s^{2}\right. \\
& +4 m^{2} r s^{2}-m^{4} r s^{2}+m^{2} r^{3} s^{2}-m s^{3} \\
& \left.+m^{3} s^{3}-m r^{2} s^{3}+2 m^{3} r^{2} s^{3}-m^{2} r s^{4}+m^{4} r s^{4}\right), \\
p_{2}= & m^{-2} s^{-3}(-1+s)(1+s)\left(r-m^{2} r+m s-m^{3} s+2 m r^{2} s\right. \\
& -m^{3} r^{2} s-r s^{2}+4 m^{2} r s^{2}-m^{4} r s^{2}+m^{2} r^{3} s^{2} \\
& \left.-m s^{3}+m^{3} s^{3}-m r^{2} s^{3}+2 m^{3} r^{2} s^{3}-m^{2} r s^{4}+m^{4} r s^{4}\right) .
\end{aligned}
$$


Neither $p_{1}$ nor $p_{2}$ are irreducible. In fact, their $G C D$ is nontrivial. Let $p=G C D\left(p_{1}\right.$, $\left.p_{2}\right)$. That is

$$
\begin{aligned}
p= & m^{2} s^{3}\left(r-m^{2} r+m s-m^{3} s+2 m r^{2} s-m^{3} r^{2} s\right. \\
& -r s^{2}+4 m^{2} r s^{2}-m^{4} r s^{2}+m^{2} r^{3} s^{2}-m s^{3} \\
& \left.+m^{3} s^{3}-m r^{2} s^{3}+2 m^{3} r^{2} s^{3}-m^{2} r s^{4}+m^{4} r s^{4}\right) .
\end{aligned}
$$

Setting $g_{1}=\frac{p_{1}}{p}=r s$ and $g_{2}=\frac{p_{2}}{p}=s^{2}-1$, we can view the representation variety as $Z\left(\left\langle g_{1} p, g_{2} p\right\rangle\right)=Z\left(\left\langle g_{1}, g_{2}\right\rangle\right) \cup Z(\langle p\rangle)$. The ideal $\left\langle g_{1}, g_{2}\right\rangle$ defines the affine variety $R_{\mathrm{a}}=\{(a, 1 / a, \pm 1, \pm 1,0)\} \subset \mathbb{C}^{6}$, which is just two copies of $\mathbb{A}^{1}$. The variety $R_{\mathrm{a}}$ is precisely all the abelian representations of $R(W)$. With the $r$ coordinate zero and the $s$ coordinate \pm 1 , any representation in $R_{\mathrm{a}}$ sends $b$ to $\pm I$. We are interested in the components of the representation variety that contain discrete faithful representations. All of these representations are in the subvariety $R=Z(\langle p\rangle)$ of $R(W)$. Hence, we are concerned with the image of $R$ under $t$ in the character variety.

The coordinate ring $T_{\mathrm{w}}$ for the Whitehead link character variety is generated by the trace maps $\left\{\tau_{a}, \tau_{b}, \tau_{a b}\right\}$. With these generators the map $t=\left(\tau_{a}, \tau_{b}, \tau_{a b}\right): R \rightarrow$ $\mathbb{C}^{3}$ is $t(\rho)=\left(m+m^{-1}, s+s^{-1}, m s+m^{-1} s^{-1}+r\right)=(x, y, z)$. Let $X^{\prime}$ denote the image of $R$ under $t$. Then the map $t: R \rightarrow X^{\prime}$ induces an injective map, $t^{*}: \mathbb{C}\left[X^{\prime}\right] \rightarrow \mathbb{C}[R]$ on the coordinates rings of $X^{\prime}$ and $R$. The coordinate ring of $R$ is $\mathbb{C}[R]=\mathbb{C}\left[m, m^{-1}, s, s^{-1}, r\right] /<p>$ so the image of $\mathbb{C}\left[X^{\prime}\right]$ under $t^{*}$ is

$$
\mathbb{C}\left[m, m^{-1}, s, s^{-1}, r\right] /\left\langle p, x=m+m^{-1}, y=s+s^{-1}, z=m s+m^{-1} s^{-1}+r\right\rangle,
$$

which is isomorphic to $\mathbb{C}[x, y, z] /\langle\tilde{f}\rangle$, where $\tilde{f}=-x y-2 z+x^{2} z+y^{2} z-x y z^{2}+z^{3}$. Since $\tilde{f}$ is smooth, $X^{\prime}$ is the affine variety $Z(\tilde{f})$. Now $X^{\prime}$ is a smooth affine surface in $\mathbb{C}^{3}$ containing the surface $\tilde{X}_{0}$. Hence $X^{\prime}=\tilde{X}_{0}$ and so $\tilde{X}_{0}$ is the hypersurface $Z(\tilde{f})$.

Throughout the rest of this section we will denote compact model $X_{0}(W)$ for the canonical component of the Whitehead link by $S$. We use the compact model obtained by taking the projective closure in $\mathbb{P}^{2} \times \mathbb{P}^{1}$. With $x, y, u$ the $\mathbb{P}^{2}$ coordinates and $z, w$ the $\mathbb{P}^{1}$ coordinates, this compactification for the canonical component $S$ is defined by $f=-w^{3} x y-2 u^{2} w^{2} z+w^{2} x^{2} z+w^{2} y^{2} z-w x y z^{2}+u^{2} z^{3}$. This surface $S$ is not smooth. It has singularities at the four points:

$$
\begin{aligned}
& s_{1}=[1,0,0,1,0], \\
& s_{2}=[0,1,0,1,0], \\
& s_{3}=[1,-1,0,1,-1], \\
& s_{4}=[1,1,0,1,1] .
\end{aligned}
$$

Our goal is to determine topologically the smooth surface $\tilde{S}$ obtained by resolving the singularities of $S$. We do this in the following theorem.

Theorem 1. The surface $\tilde{S}$ is a rational surface isomorphic to $\mathbb{P}^{2}$ blown up at ten points.

The Euler characteristic of $\tilde{S}$ together with the fact that $\tilde{S}$ is rational is enough to determine $\tilde{S}$ up to isomorphism. 
Lemma 1. $\tilde{S}$ is birational to a conic bundle.

Proof. Consider the projection $\pi_{\mathbb{P}^{1}}: S \rightarrow \mathbb{P}^{1}$. The fiber over $\left[z_{0}, w_{0}\right] \in \mathbb{P}^{1}$ is the set of points $\left[x, y, u: z_{0}, w_{0}\right]$, which satisfy $-w_{0}^{3} x y-2 u^{2} w_{0}^{2} z_{0}+w_{0}^{2} x^{2} z_{0}+w_{0}^{2} y_{0}^{2} z-w_{0} x y z_{0}^{2}+$ $u^{2} z_{0}^{3}=0$. This is the zero set of a degree 2 polynomial in $\mathbb{P}^{2}$, which is a conic. Away from the four singularities, $S$ is isomorphic to a conic bundle. Hence, $S$ is birational to a conic bundle. Since $\tilde{S}$ is obtained from $S$ by a series of blow ups, $\tilde{S}$ is birational to $S$ and so birational to a conic bundle.

Applying Proposition 1 we now have that $\tilde{S}$ is rational surface. Since $S$ has degenerate fibers, $\tilde{S}$ is not isomorphic to $\mathbb{P}^{1} \times \mathbb{P}^{1}$ (see Figure 2). So, by Corollary 1 $\tilde{S}$ is topologically $\mathbb{P}^{2}$ blown up at $n$ points. It follows from Proposition 2 that $\chi(\tilde{S})=\chi\left(\mathbb{P}^{2}\right)+n=3+n$. Thus, we can determine $n$ from the Euler characteristic of $\tilde{S}$.

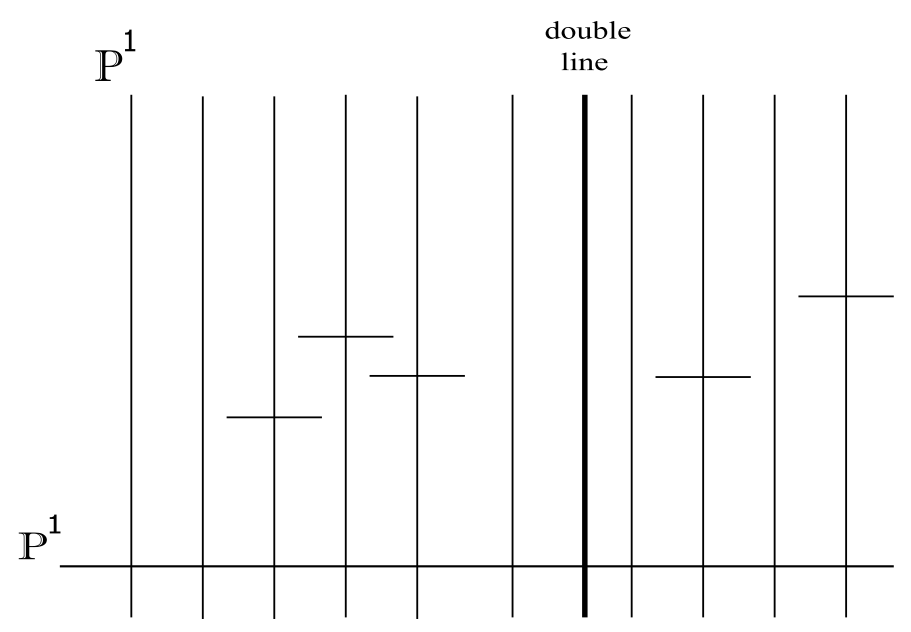

Figure 2. Canonical component of the Whitehead link.

To calculate the Euler characteristic of $\tilde{S}$, we use the Euler characteristic of $S$. Since the smooth surface $\tilde{S}$ is obtained from $S$ by a series of blow ups, we can use Proposition 3 to write $\chi(\tilde{S})$ in terms of $\chi(S)$.

Lemma 2. $\chi(\tilde{S})=\chi(S)+4$.

Proof. The smooth surface, $\tilde{S}$, is obtained by resolving the four singularities, $s_{i}$, of $S$ listed above. Above the singularities, a local model for $\tilde{S}$ can be obtained by blowing up $S$ in an affine neighborhood of each of the singular points. Away from the singularities we can take the local model for $S$ as a local model for $\tilde{S}$ since $S$ and $\tilde{S}$ are locally isomorphic there. Each of the singularities is nice in the sense that it takes only one blow up to resolve them. Hence, in terms of the Euler characteristic, we have

$$
\chi(\tilde{S})=\chi\left(S-\left\{s_{i}\right\}\right)+\Sigma_{i=1}^{4} \chi\left(\tilde{s}_{i}\right),
$$

where for $i=1 \ldots 4, \tilde{s_{i}}$ denotes the preimage of $s_{i}$ in $\tilde{S}$. Determining the Euler characteristic of $\tilde{S}$ in terms of that for $S$ reduces to determining $\tilde{s_{i}}$. 
To blow up $S$ at $s_{1}=[1,0,0,1,0]$ we consider the affine open set $A_{1}^{\prime}$ where $x \neq 0$ and $z \neq 0$. Noticing that the singularities $s_{3}$ and $s_{4}$ are in $A_{1}^{\prime}$, we look at the blow up of $S$ at $s_{1}$ in the affine open set $A_{1}=A_{1}^{\prime} \backslash\left\{s_{3}, s_{4}\right\}$. Local affine coordinates for $A_{1} \cong \mathbb{A}^{3}$ are $y, u, w$. So to blow up $S$ at $s_{1}$ we blow up $X_{1}=Z\left(\left.f\right|_{x=1, z=1}\right)$ at $[y, u, w]=[0,0,0]$ in $A_{1}$. As described in Section 3.2, the blow up of $X_{1}$ at $[0,0,0]$ is the closure of the preimage of $X_{1}-[0,0,0]$ in $\left.B l\right|_{[0,0,0]}\left(A_{1}\right)$. Using coordinates $a, b, c$ for $\mathbb{P}^{2}$, the blow up $Y_{1}$ of $X_{1}$ at $[0,0,0]$ is the closed subset in $A_{1} \times \mathbb{P}^{2}$ defined by the equations

$$
\begin{aligned}
f_{1}=\left.f\right|_{x=1, z=1} & =u^{2}+w^{2}-2 u^{2} w^{2}-w y-w^{3} y+w^{2} y^{2}, \\
e_{1} & =y b-u a \\
e_{2} & =y c-w a \\
e_{3} & =u c-w b .
\end{aligned}
$$

We determine the local model above $s_{1}$ and check for smoothness by looking at $Y_{1}$ in the affine open sets define by $a \neq 0, b \neq 0$, and $c \neq 0$.

First, we look at $Y_{1}$ in the affine open set defined by $a \neq 0$ (i.e. we can set $a=1$ ). In this open set the defining equations for $Y_{1}$ become

$$
\begin{aligned}
& f_{1}=u^{2}+w^{2}-2 u^{2} w^{2}-w y-w^{3} y+w^{2} y^{2}, \\
& e_{1}=y b-u \\
& e_{2}=y c-w \\
& e_{3}=u c-w b .
\end{aligned}
$$

Using equations $e_{1}$ and $e_{2}$ and substituting for $u$ and $w$ in $f_{1}$, we obtain the local model, $y^{2}\left(-b^{2}+c-c^{2}-c^{2} y^{2}+2 b^{2} c^{2} y^{2}+c^{3} y^{2}\right)$. The first factor is the exceptional plane, $E_{1}$ and the other factor is the local model for $Y_{1}$. Notice that $E_{1}$ and $Y_{1}$ meet in the smooth conic $-b^{2}+c-c^{2}$. So, in this affine open set, the local model above the singularity $s_{1}$ is a conic, and therefore isomorphic $\mathbb{P}^{1}$. Since the only places all the partial derivatives of the second factor vanish are over the singular points $s_{3}$ and $s_{4}$, this model is smooth in $A_{1} \times \mathbb{P}^{2}$.

Next we look at $Y_{1}$ in the affine open set defined by $b \neq 0$. In this open set, the defining equations for $Y_{1}$ become

$$
\begin{aligned}
& f_{1}=u^{2}+w^{2}-2 u^{2} w^{2}-w y-w^{3} y+w^{2} y^{2}, \\
& e_{1}=y-u a \\
& e_{2}=y c-w a \\
& e_{3}=u c-w .
\end{aligned}
$$

Substituting into $f_{1}$, we obtain the local model, $u^{2}\left(1-a c+c^{2}-2 c^{2} u^{2}+a^{2} c^{2} u^{2}-a c^{3} u^{2}\right)$. Again, the first factor is the exceptional plane, $E_{1}$ and the other factor is the local model for $Y_{1}$. Notice that $E_{1}$ and $Y_{1}$ meet in the smooth conic $1-a c+c^{2}$. So, in this affine open set, the local model above the singularity $s_{1}$ is a conic. Since all the partial derivatives of the second factor do not simultaneously vanish, this model is smooth in $A_{1} \times \mathbb{P}^{2}$. 
Finally, we look at $Y_{1}$ in the affine open set defined by $c \neq 0$. In this open set the defining equations for $Y_{1}$ become

$$
\begin{aligned}
& f_{1}=u^{2}+w^{2}-2 u^{2} w^{2}-w y-w^{3} y+w^{2} y^{2} \\
& e_{1}=y b-u a \\
& e_{2}=y-w a \\
& e_{3}=u-w b .
\end{aligned}
$$

Substituting into $f_{1}$, we obtain the local model, $w^{2}\left(1-a+b^{2}-a w^{2}+a^{2} w^{2}-2 b^{2} w^{2}\right)$. The first factor is the exceptional plane, $E_{1}$ and the other factor is the local model for $Y_{1}$. Notice that $E_{1}$ and $Y_{1}$ meet in the smooth conic $1-a+b^{2}$. So, in this affine open set, the local model above the singularity $s_{1}$ is a conic. Since the only places all the partial derivatives of the second factor vanish simultaneously are $s_{3}$ and $s_{4}$, this model is smooth in $A_{1} \times \mathbb{P}^{2}$.

Rehomogenizing we see that blowing up yields a smooth local model that intersects the exceptional plane above $s_{1}$ in the conic defined by $c^{2}-a+b^{2}$. Hence $\chi\left(\tilde{s_{1}}\right)=2$.

Blowing up $S$ at $s_{2}, s_{3}$ and $s_{4}$ is similar to blowing up $S$ at $s_{1}$. For detailed calculations, we refer the reader to the author's thesis.

In each case local model for $\left.B l(S)\right|_{s_{i}}$ intersects the exceptional plane above $s_{i}$ in a smooth conic. Hence $\chi\left(\tilde{s}_{i}\right)=2$ for $i=1, \ldots, 4$ and

$$
\begin{aligned}
\chi(\tilde{S}) & =\chi\left(S-\left\{s_{i}\right\}\right)+\Sigma_{i=1}^{4} \chi\left(\tilde{s}_{i}\right), \\
& =\chi(S)-\Sigma_{i=1}^{4} \chi\left(s_{i}\right)+\Sigma_{i=1}^{4} \chi\left(\tilde{s}_{i}\right), \\
& =\chi(S)-4+4(2), \\
& =\chi(S)+4 .
\end{aligned}
$$

Proposition 5. The Euler characteristic of the surface $S$ is $\chi(S)=9$.

To calculate the Euler characteristic we will appeal to the map $\phi: S \rightarrow \mathbb{P}^{1} \times \mathbb{P}^{1}$ defined by $[x, y, u: z, w] \rightarrow[x, y: z, w]$ on a dense open subset of $\mathrm{S}$. That the map $\phi$ is generically 2 -to- 1 makes it an attractive tool in determining the Euler characteristic of $S$. However, in order to calculate the Euler characteristic of $S$ we must understand the map $\phi$ everywhere not just generically. To this affect, there are four aspects we need to consider. The map $\phi$ is neither surjective nor defined at the three points $P=\left\{(0,0,1,0,1),\left(0,0,1,1, \pm \frac{1}{\sqrt{2}}\right)\right\}$. Over six points in $\mathbb{P}^{1} \times \mathbb{P}^{1}$ the fiber is a copy of $\mathbb{P}^{1}$. Finally, the map is branched over three copies of $\mathbb{P}^{1}$. We explain how to alter the Euler characteristic calculation to account for each of these situations.

Lemma 3. The image of $\phi$ on $U=S-P$ is $\mathbb{P}^{1} \times \mathbb{P}^{1}-Q$ where

$$
\begin{aligned}
Q & =\mathbb{P}^{1} \times\{[0,1]\} \backslash\{[1,0,0,1],[0,1,0,1]\} \\
& \cup \mathbb{P}^{1} \times\left\{\left[1, \frac{1}{\sqrt{2}}\right]\right\} \backslash\left\{\left[\frac{1}{\sqrt{2}}, 1,1, \frac{1}{\sqrt{2}}\right],\left[\sqrt{2}, 1,1, \frac{1}{\sqrt{2}}\right]\right\} \\
& \cup \mathbb{P}^{1} \times\left\{\left[1,-\frac{1}{\sqrt{2}}\right]\right\} \backslash\left\{\left[-\frac{1}{\sqrt{2}}, 1,1,-\frac{1}{\sqrt{2}}\right],\left[-\sqrt{2}, 1,1,-\frac{1}{\sqrt{2}}\right]\right\} .
\end{aligned}
$$

Proof. We can see that this is in fact the image by viewing $f$ as a polynomial in $u$ with coefficients in $\mathbb{C}[x, y, z, w]$. Namely $f=g+u^{2} h$ where $g=-w^{3} x y+w^{2} x^{2} z+$ 
$w^{2} y^{2} z-w x y z^{2}$ and $h=z\left(z^{2}-2 w^{2}\right)$. The image of $\phi$ is the collection of all points $[x, y, z, w] \in \mathbb{P}^{1} \times \mathbb{P}^{1}$ except those for which $f(x, y, z, w) \in \mathbb{C}[u]$ is a nonzero constant. The polynomial $f(x, y, z, w)$ is a nonzero constant, whenever $h=0$ and $g \neq 0$. It is easy to see that $h=0$, whenever $[z, w]=\left\{[0,1],\left[1, \pm \frac{1}{\sqrt{2}}\right]\right\}$. For each of the $z, w$ coordinates that satisfy $h$, there are two $x, y$ coordinates that satisfy $g(z, w)$. Hence the image of $\phi$ on $U$ is all of $\mathbb{P}^{1} \times \mathbb{P}^{1}$ less the three twice punctured spheres as listed above.

Lemma 4. The map $\phi$ smoothly extends to all of $S$.

Proof. We can extend the map $\phi$ to all of $S$ by using a total transformation. Let $U=S-P$. Then $U$ is the largest open set in $S$ on which $\phi$ is defined. Let $\overline{G(\phi, U)}$ be the closure of the graph of $\phi$ on $U$. We can then smoothly extend the map $\phi$ to all of $S$ by defining $\phi$ at each $p_{i} \in P$ to be $\phi\left(p_{i}\right):=\rho_{2} \rho_{1}^{-1}\left(p_{i}\right)$ where $\rho_{1}: \bar{G} \rightarrow S$ and $\rho_{2}: \bar{G} \rightarrow \mathbb{P}^{1} \times \mathbb{P}^{1}$ are the natural projections. Note that, for $s \in U, \rho_{2} \rho_{1}^{-1}(s)$ coincides with the original map so that this extension makes sense on all of $S$. Now, the closure of the graph is $\bar{G}=\{[x, y, u, z, w: a, b, c, d] \mid f=0, a y=b x, c w=d z\}$. So, $\phi$ extends to $S$ as follows:

$$
\begin{aligned}
\phi((0,0,1,0,1)) & =\{[a, b, 0,1]\}, \\
\phi\left(\left(0,0,1,1, \frac{1}{\sqrt{2}}\right)\right) & =\left\{\left(a, b, 1, \frac{1}{\sqrt{2}}\right)\right\}, \\
\phi\left(\left(0,0,1,1,-\frac{1}{\sqrt{2}}\right)\right) & =\left\{\left(a, b, 1,-\frac{1}{\sqrt{2}}\right)\right\} .
\end{aligned}
$$

Notice that the set $Q \subset \mathbb{P}^{1} \times \mathbb{P}^{1}$, which is not contained in the image of $\phi$ on $U$, is contained in the image of $\phi$ on $P$. That the extension $\phi$ maps three points in $S$ to not just three disjoint $\mathbb{P}^{1}$ 's in $\mathbb{P}^{1} \times \mathbb{P}^{1}$ but to the three disjoint $\mathbb{P}^{1}$ 's which are are missing from the image of $\phi$ on $U$ will be important for the Euler characteristic calculation.

Lemma 5. There are six points in $\mathbb{P}^{2} \times \mathbb{P}^{1}$, the collection of which we will call $L$, whose fiber in $S$ is infinite.

Proof. Thinking of $f$ as a polynomial in the variable $u$ with coefficients in $\mathbb{C}[x, y, z, w]$, we see that the points in $\mathbb{P}^{1} \times \mathbb{P}^{1}$, which are simultaneously zeros of these coefficient polynomials are precisely the points in $\mathbb{P}^{1} \times \mathbb{P}^{1}$ whose fiber is infinite. We note here that the points of $L$ are precisely the punctures of the three punctured spheres that are not in the image of $\left.\phi\right|_{U}$. The preimage of $L$ in $\mathrm{S}$ is the union of six $\mathbb{P}^{1}$ 's each intersecting exactly one other $\mathbb{P}^{1}$ in one point. These three points of intersection are the points on the $\mathbb{P}^{1}$ 's where the coordinate $u$ goes to infinity which is equivalent to the points where the $x$ and $y$ coordinates go to zero. Thus these intersection points are precisely the points in $P$. The points in $L$ along with their infinite fibers in $\mathbb{P}^{1} \times \mathbb{P}^{1}$ are listed below.

$$
\begin{array}{lll}
{[1,0,0,1]} & \text { has fiber } & \{[1,0, u, 0,1]\} \supset[0,0,1,0,1], \\
{[0,1,0,1]} & \text { has fiber } & \{[0,1, u, 0,1]\} \supset 0,0,1,0,1], \\
{\left[1, \sqrt{2}, 1, \frac{1}{\sqrt{2}}\right]} & \text { has fiber } & \left\{\left[1, \sqrt{2}, u, 1, \frac{1}{\sqrt{2}}\right] \supset \supset 0,0,1,1, \frac{1}{\sqrt{2}}\right], \\
{\left[1, \frac{1}{\sqrt{2}}, 1, \frac{1}{\sqrt{2}}\right]} & \text { has fiber } & \left\{\left[1, \frac{1}{\sqrt{2}}, u, 1, \frac{1}{\sqrt{2}}\right]\right\} \subset\left[0,0,1,1, \frac{1}{\sqrt{2}}\right], \\
{\left[1,-\sqrt{2}, 1,-\frac{1}{\sqrt{2}}\right]} & \text { has fiber } & \left\{\left[1,-\sqrt{2}, u, 1,-\frac{1}{\sqrt{2}}\right]\right\} \supset\left[0,0,1,1,-\frac{1}{\sqrt{2}}\right], \\
{\left[1,-\frac{1}{\sqrt{2}}, 1,-\frac{1}{\sqrt{2}}\right]} & \text { has fiber } & \left\{\left[1,-\frac{1}{\sqrt{2}}, u, 1,-\frac{1}{\sqrt{2}}\right]\right\} \supset\left[0,0,1,1,-\frac{1}{\sqrt{2}}\right]
\end{array}
$$


In calculating the Euler characteristic we will use the fact that the preimage of $L$ in $S$ is six $\mathbb{P}^{1}$ 's which intersect in pairs at ideal points in the set $P \subset S$. In fact, each point in $P$ appears as the intersection of two of these fibers and the image of $P$ under $\phi$ is precisely $L$.

Let $B$ denote the branch set of $\phi$ in $\mathbb{P}^{1} \times \mathbb{P}^{1}$. We have the following lemma.

Lemma 6. $\chi(B)=2$.

Proof. The branch set, or at least the places where $\phi$ is not one-to-one, consists of the points in $S$ which also satisfy the coordinate equation $u=0$. The image, $B \subset \mathbb{P}^{1} \times \mathbb{P}^{1}$, of this branch set, is the union of the three varieties, $B_{1}, B_{2}$, and $B_{3}$ defined by the respective three polynomials $f_{1}=w y-x z, f_{2}=w x-y z$, and $f_{3}=w$ which are all $\mathbb{P}^{1}$ 's. From the bidegrees of the $f_{i}$ we know that $B_{3}$ intersects each of $B_{1}$ and $B_{2}$ in one point $\left([0,1,1,0]\right.$ and $[1,0,1,0]$, respectively), while $B_{1}$ and $B_{2}$ intersect in two points $([1,-1,-1,1]$ and $[1,1,1,1])$. Again thinking of $f$ as a polynomial in $u$, we can write $f$ as $f=A+u^{2} B$ where $A$ and $B$ are polynomials in $\mathbb{C}[x, y, z, w]$. Since $L$ is cut out by the ideal $\langle A, B\rangle$ and $\mathrm{B}$ is cut out by the ideal $\langle A\rangle, L$ is a subvariety of $B$. That each of six points in $\mathbb{P}^{1} \times \mathbb{P}^{1}$ whose fiber is infinite is also a branch point is necessary for the Euler characteristic calculation.

Now that we understand the map $\phi$ everywhere we can calculate the Euler characteristic of $S$ and prove Proposition 5 .

Proof (Proposition 5). Since the set of points in $\mathbb{P}^{1} \times \mathbb{P}^{1}$ whose fibers are infinite coincide with the image $L$ of the fundamental set $P$, and $L$ is the intersection of $Q$ and the branch set $B$,

$$
\begin{aligned}
\chi(s) & =2 \chi\left(\mathbb{P}^{1} \times \mathbb{P}^{1}-B-Q\right)+\chi(Q+B-L)+\chi\left(\phi^{-1}(L)\right) \\
& =2 \chi\left(\mathbb{P}^{1} \times \mathbb{P}^{1}\right)-\chi(Q)-\chi(B)-\chi(L)+\chi\left(\phi^{-1}(L)\right) .
\end{aligned}
$$

The Euler characteristic of $\mathbb{P}^{1} \times \mathbb{P}^{1}$ is $\chi\left(\mathbb{P}^{1} \times \mathbb{P}^{1}\right)=4$. As $Q$ is the disjoint union of three twice-punctured spheres, $\chi(Q)=3\left(\chi\left(\mathbb{P}^{1}\right)-2\right)=0$. Since $B$ is three $\mathbb{P}^{1}$ 's which intersect at four points, $\chi(B)=3 \chi\left(\mathbb{P}^{1}\right)-4 \chi($ point $)=2$. Now $L$ is just six points so $\chi(L)=6$. That $\phi^{-1}(L)$ is the union of six $\mathbb{P}^{1}$ 's which intersect in pairs at a point implies that $\chi\left(\phi^{-1}(L)\right)=6 \chi\left(\mathbb{P}^{1}\right)-3 \chi($ points $)=9$. All together this gives $\chi(S)=9$.

Corollary 2. The Euler characteristic of $\tilde{S}$ is $\chi(\tilde{S})=13$.

Proof. We have $\chi(\tilde{S})=\chi(S)+4=9+4=13$.

We are now ready to prove Theorem 1.

Proof (Theorem 1). It follows from Lemma 1, Corollary 1, and Proposition 2 that $\chi(\tilde{S})=\chi\left(\mathbb{P}^{2}\right)+n$. By Corollary $2, n$ must be 10 and $\tilde{S}$ must be $\mathbb{P}^{2}$ blown up at 10 points. 


\section{Other two component 2-bridge link examples}

The Whitehead link complement can be obtained by $1 / 1$ Dehn surgery on the Borromean rings (Figure 3), the complement of which we will denote by $M_{\mathrm{br}}$.

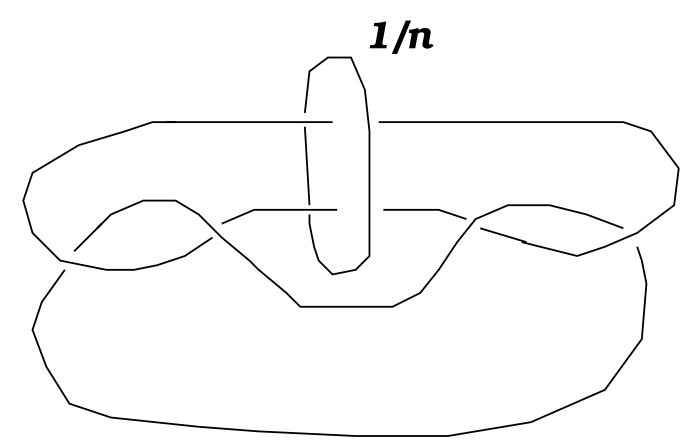

FiguRE 3. $1 / n$ Dehn surgery on the Borromean rings.

The manifolds that result from $1 / n$ Dehn filling on one of the cusps of $M_{\mathrm{br}}$ are [6] two component 2-bridge links with Schubert normal form $S(8 n, 4 n+1)$ (Figure 3). The fundamental group of these two component 2-bridge links has a presentation of the form $\Gamma=\langle a, b \mid a w=w a\rangle$ with $w=b^{\epsilon_{1}} a^{\epsilon_{2}} \ldots b^{\epsilon_{8 n-1}}$, where $\epsilon_{i}=(-1)^{\left\lfloor\frac{i(4 n-1)}{8 n}\right\rfloor}$. For $n=1, \ldots, 4$, we were able to use Mathematica to determine the polynomials that define the character varieties of $M_{\mathrm{br}}(1 / n)$. For $n \geq 4$ the polynomials are a bit too large for Mathematica to handle.

Although we have few examples, there are some trends among these character varieties, which make them worth noting. We can look at the number of components and the number of canonical components that comprise these character varieties. Although the defining polynomials for certain components may not be smooth, their bidegree and how they change with the surgery coefficient $n$ is still of interest. Below we summarize this information (Table 1).

TABLE 1. Character varieties for $M_{\mathrm{br}}(1 / n)$.

\begin{tabular}{cccc}
\hline Manifold & Component & Canonical component & Bidegree \\
\hline$M_{\mathrm{br}}(1 / 1)$ & 1 & $\sqrt{ }$ & $(2,3)$ \\
\hline$M_{\mathrm{br}}(1 / 2)$ & 1 & & $(2,2)$ \\
\hline & 2 & & $(4,5)$ \\
\hline$M_{\mathrm{br}}(1 / 3)$ & 1 & & $(2,2)$ \\
\hline & 2 & & $(2,2)$ \\
\hline & 3 & & $(6,7)$ \\
\hline$M_{\mathrm{br}}(1 / 4)$ & 1 & $\sqrt{ }$ & $(2,2)$ \\
\hline & 2 & & $(4,4)$ \\
\hline & 3 & & $(8,9)$ \\
\hline
\end{tabular}

Possibly the most interesting characteristic these character varieties share is the existence of a component, which is defined by a polynomial of bidegree $(2, k)$ where 
$k=\{2,3\}$. All of these components are $\mathbb{P}^{1}$ bundles over $\mathbb{P}^{1}$. Although they are not conic bundles due to the existence of singularities, they all share a common feature. Over the same $\mathbb{P}^{1}$ coordinate $([z, w]=[1,0])$, they all have a double line fiber. See Figure 4.

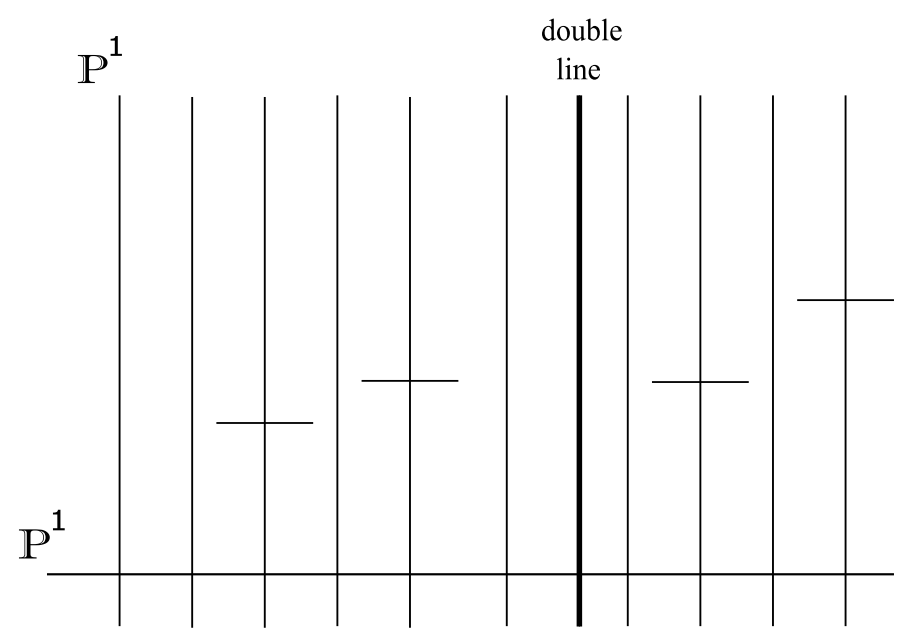

Figure 4. Component birational to a conic bundle for $M_{\mathrm{br}}(1 / n)$.

Away from a few points all of these components look like conic bundles. All conics are parameterized by $\mathbb{P}^{5}$ and so we can think of conic bundles as curves in $\mathbb{P}^{5}$. All the degeneracies live in a hypersurface in $\mathbb{P}^{5}$ and all the double lines live in a codimension 2 subvariety inside this hypersurface. Hence, it is fairly uncommon for a curve in $\mathbb{P}^{5}$ to intersect the subvariety which corresponds to double line fibers.

All of these components are defined by polynomials that have singularities (four for the Whitehead link and two for each of the other components). While these $\mathbb{P}^{1}$ bundles are not isomorphic to conic bundles, they are birational to such. Since surfaces birational to conic bundles are rational, all of these components are rational surfaces and thus isomorphic either to $\mathbb{P}^{1} \times \mathbb{P}^{1}$ or $\mathbb{P}^{2}$ blown up at some number of points. As we did for the Whitehead link complement, we can use the Euler characteristic to determine these character variety components topologically. Aside from the Whitehead link all of these components of these character varieties are hypersurfaces defined by a singular polynomial of bidegree $(2,2)$ in $\mathbb{P}^{2} \times \mathbb{P}^{1}$. Each of these defining polynomials has two singularities that each resolve into a conic after a single blow up. Hence the Euler characteristic of the smooth models are equal to that of the singular models plus two. The way we calculate the Euler characteristic of these singular models is very similar to way to we calculate such for the Whitehead link. It turns out that all of these singular (and so smooth) models have Euler characteristic 10 and hence are all isomorphic to $\mathbb{P}^{2}$ blown up at 7 points. From an algebro-geometric perspective $\mathbb{P}^{2}$ blown up at seven points is interesting in the sense that is has only finitely many (precisely 49) (-1) curves.

What we have just described is a brief outline of the proof of Theorem 2 . 
Theorem 2. For $n=2,3,4$, the character variety of $M_{b r}(1 / n)$ has a component which is a rational surface isomorphic to $\mathbb{P}^{2}$ blown up at seven points.

The proof is very similar to that for Theorem 1. Hence, we omit the details here. Below we have listed the singular defining polynomials for these conic bundle components (Table 2).

TABLE 2. Conic bundle components for $M_{\mathrm{br}}(1 / n)$.

\begin{tabular}{llll}
\hline Manifold & $\begin{array}{l}\text { Singular defining polyno- } \\
\text { mial for conic bundle } \\
\text { component }\end{array}$ & $\begin{array}{l}\text { Euler } \\
\text { characteristic }\end{array}$ & $\begin{array}{l}\text { Smooth complex } \\
\text { surface }\end{array}$ \\
\hline$M_{\mathrm{br}}(1 / 1)$ & $\begin{array}{l}-w^{3} x y+w^{2} x^{2} z+w^{2} y^{2} z- \\
w x y z^{2}+u^{2}\left(z^{3}-2 w^{2} z\right)\end{array}$ & 13 & $\mathbb{P}^{2}$ blown up at 10 points \\
\hline$M_{\mathrm{br}}(1 / 2)$ & $\begin{array}{l}w^{2} x^{2}+w^{2} y^{2}-w x y z+ \\
u^{2}\left(z^{2}-2 w^{2}\right)\end{array}$ & 10 & $\mathbb{P}^{2}$ blown up at 7 points \\
\hline$M_{\mathrm{br}}(1 / 3)$ & $w^{2} x^{2}+w^{2} y^{2}-w x y z+$ & 10 & $\mathbb{P}^{2}$ blown up at 7 points \\
& $u^{2}\left(z^{2}-3 w^{2}\right)$ & & \\
\hline & $w^{2} x^{2}+w^{2} y^{2}-w x y z+$ & 10 & $\mathbb{P}^{2}$ blown up at 7 points \\
& $u^{2}\left(z^{2}-w^{2}\right)$ & & \\
\hline$M_{\mathrm{Fbr}}(1 / 4)$ & $w^{2} x^{2}+w^{2} y^{2}-w x y z+$ & 10 & $\mathbb{P}^{2}$ blown up at 7 points \\
& $u^{2}\left(z^{2}-2 w^{2}\right)$ & & \\
\hline
\end{tabular}

\section{References}

[1] A. Beauville, Complex algebraic surfaces, volume 34 of London Mathematical Society Student Texts, Cambridge University Press, Cambridge, 2nd ed., 1996. Translated from the 1978 French original by R. Barlow, with assistance from N. I. Shepherd-Barron and M. Reid.

[2] M. Culler, C. M. Gordon, J. Luecke and P. B. Shalen, Dehn surgery on knots [Ann. of Math. (2) 125(2) (1987), 237-300; MR0881270 (88a:57026)]. Ann. Math. (2) 127(3) (1988), 663.

[3] M. Culler and P. B. Shalen, Varieties of group representations and splittings of 3-manifolds, Ann. Math. (2) 117(1) (1983), 109-146.

[4] P. Griffiths and J. Harris, Principles of algebraic geometry, Wiley Classics Library. John Wiley \& Sons Inc., New York, 1994. Reprint of the 1978 original.

[5] R. Hartshorne, Algebraic geometry, in "Graduate texts in mathematics", Springer-Verlag, New York, NY, 1977.

[6] J. Hoste and P. D. Shanahan, Computing boundary slopes of 2-bridge links, Math. Comput. 76(259) (2007), 1521-1545 (electronic).

[7] M. L. Macasieb, K. L. Petersen and R. M. van Luijk, On character varieties of two-bridge knot groups, to appear in Proc. LMS.

[8] I. R. Shafarevich, Basic algebraic geometry, 1. Springer-Verlag, 2nd ed., Varieties in projective space; Translated from the 1988 Russian ed. and with notes by Miles Reid.

[9] P. B. Shalen, Representations of 3-manifold groups, in "Handbook of geometric topology", North-Holland, Amsterdam, 2002, 955-1044.

[10] W. P. Thurston, Three-dimensional geometry and topology, volume 35 of Princeton Mathematical Series, 1, (ed. Silvio Levy), Princeton University Press, Princeton, NJ, 1997.

Mathematics Department, University of Texas at Austin, 1 University Station C1200 Austin, TX 78712, USA

E-mail address: elandes@math.utexas.edu 
